\title{
LOCALIZATIONS AND EVALUATION SUBGROUPS
}

GEORGE E. LANG, JR.

ABSTRACT. If $G_{n}(X)$ is the $n$th evaluation subgroup of a simple connected finite $C W$-complex, then $G_{n}\left(X_{p}\right) \cong G_{n}(X)_{p}$ for $p=0$ or a prime.

Let $X$ be a connected simple finite $C W$-complex, $X_{p}$ its localization at $p$ for $p$ prime or 0 [5], and $G_{n}(X)$ the evaluation subgroup of $\pi_{n}(X)[1]$. If $e_{p}: X \rightarrow X_{p}$ is the canonical map, we show that $\alpha \in G_{n}(X)$ if and only if $e_{p^{*}}(\alpha) \in G_{n}\left(X_{p}\right)$ for all $p$. As corollaries we obtain that $G_{n}\left(X_{p}\right) \cong G_{n}\left(X_{p}\right)$ and $X$ is a $G$-space [1] if and only if $X_{p}$ is a $G$-space for all $p$, where $G_{n}(X)_{p}$ is the localization of the group $G_{n}(X)$. This is analogous to results obtained for $H$-spaces [3], [5]. All $H$-spaces are $G$-spaces, and many properties of $H$-spaces are shared by $G$-spaces.

1. Preliminaries. Spaces $X$ and $W$ are assumed to be pointed, simple (abelian fundamental groups acting trivially on the homotopy and homology groups), connected, finite $C W$-complexes. We will not distinguish between a map and its homotopy class. For $p$ a prime let $Q_{p}=\{k / q \mid k, q$ integers, $p \nmid q\}$ and $Q_{0}$ the rationals. $Q_{p}$ is the localization of the integers at the prime p. The general reference for localization theory is [5]. We review some of these results here.

Definition 1.1. A space $X$ is $p$-local if $\pi_{*}(X)$ admits a $Q_{p}$-module structure extending the usual $Z$-module structure.

For each $X$ there is a $p$-local space $X_{p}$ and canonical map $e_{p}: X \rightarrow X_{p}$ such that if $g: X \rightarrow Y$, where $Y$ is $p$-local, there is a unique (up to homotopy) $g^{\prime}: X_{p} \rightarrow Y$ such that $g \simeq g^{\prime} e_{p}$. This is equivalent to the map $\phi_{p}: \pi_{n}(X) \otimes Q_{p}$ $\rightarrow \pi_{n}\left(X_{p}\right)$ being an isomorphism, where $\phi_{p}(\alpha \otimes r)=r e_{p} *(\alpha)$, where the multiplication is the $Q_{p}$-module structure on $\pi_{n}\left(X_{p}\right)\left(e_{p} p\right.$-localizes in homotopy). Finally we point out that localization is functorial.

Key results about the evaluation subgroups can be found in [1]. We will establish the notations needed in this paper. $L(W, X ; f)$ will be the

Presented to the Society, January 26, 1975 under the title Localizations and $G_{n}(X)$; received by the editors July 2, 1973 and, in revised form, February 27, 1974.

AMS (MOS) subject classifications (1970). Primary 55E05; Secondary 55F05.

Key words and phrases. Localization, evaluation subgroup. 
path component containing $f: W \rightarrow X$ in the space of functions from $W$ to $X$ with the compact-open topology; $L_{0}(W, X ; f)$ will be the subspace of $L(W, X ; f)$ containing base point preserving functions; $\omega: L(W, X ; f) \rightarrow X$ will be the function which evaluates each map at the base point of $W$.

Definition 1.2. The evaluation subgroup, $G_{n}(X)$, of $\pi_{n}(X)$ is defined by

$$
G_{n}(X)=\operatorname{Im}\left(\omega_{*}: \pi_{n}\left(L\left(X, X ; 1_{X}\right)\right) \rightarrow \pi_{n}(X)\right)
$$

where $1_{X}$ is the identity on $X$.

$G_{n}(X)$ consists of all elements $\alpha \in \pi_{n}(X)$ such that there is a function $F: X \times S^{n} \rightarrow X$ with $F \mid X \vee S^{n}=1_{X} \vee \alpha$. Such a function will be called an affilated map for $\alpha$.

2. Localizations and evaluation subgroups. Let

$$
\hat{e}_{p}: L(W, X: f) \rightarrow L\left(W, X_{p} ; e_{p} f\right)
$$

be defined by $\hat{e}_{p}(g)=e_{p} g$.

Theorem 2.1. If $W$ is a connected finite CW-complex and $X$ a connected simple CW-complex then (1) p-localizes in homotopy.

Proof. By Corollary 1.3 in [3] $\bar{e}_{p}=\hat{e}_{p} \mid L_{0}(W, X ; f) p$-localizes. Consider the following commutative diagram:

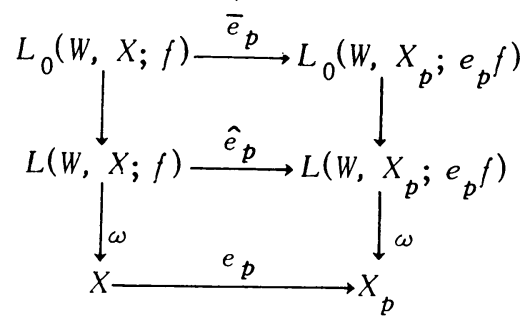

Since $\omega$ is a fibration in both columns and two of the three horizontal maps $p$-localize, $\hat{e}_{p}$ also $p$-localizes (see 2.21 and [5]).

Theorem 2.2. If $\alpha \in G_{n}(X)$ then $e_{p} *(\alpha) \in G_{n}\left(X_{p}\right)$.

Proof. Let $F: X \times S^{n} \rightarrow X$ be an affiliated map for $\alpha$. Let $\bar{F}$ be the composition

$$
X_{p} \times S^{n} \stackrel{1 \times e_{p}}{\longrightarrow} X_{p} \times S_{p}^{n} \stackrel{F_{p}}{\longrightarrow} X_{p} .
$$

Note that $\left(X \times S^{n}\right)_{p}=X_{p} \times S_{p}^{n}$. Since $\bar{F} \mid S^{n}=\alpha_{p} e_{p}=e_{p} \alpha, \bar{F}$ is an affiliated map for $e_{p}(\alpha)$. This result does not require $X$ to be finite.

Theorem 2.3. If $e_{p^{*}}(\alpha) \in G_{n}\left(X_{p}\right)$ for all $p$ then $\alpha \in G_{n}(X)$. 
Proof. Case 1. a of finite order. Let $p_{1}^{m} 1_{p_{2}}^{m_{2}} \cdots p_{n}^{m}$ be the prime factorization of the order $a$. Let $P_{1}=\prod_{i \neq 1} p_{1}^{m_{1}}$. Since $p_{1}^{m_{1}}$ and $P_{1}$ are relatively prime, there are integers $r$ and $s$ such that $1=r p_{1}{ }_{1}{ }_{1}+s P_{1}$; so $\alpha=r p_{1}^{m} \alpha+s P_{1} a . s P_{1} \alpha$ is of order $p_{1}^{m_{1}}$ while $r p_{1}^{m_{1}} \alpha$ is of order $P_{1}$. $r p_{1}^{m}{ }^{1} \alpha$ can then be written as a sum of its (hence $\alpha$ 's) multiples such that one summand is of order $p_{2}^{m_{2}}$ and the other of order $\prod_{m_{i}}>{ }_{2} p_{i}^{m_{i}}$. By induction $\alpha=k_{1} \alpha+k_{2} \alpha+\cdots+k_{n} \alpha$ where $k_{i} \alpha$ is of order $p_{i}^{m_{i}}$. Since $e_{p^{*}}(\alpha) \epsilon$ $G_{n}\left(X_{p}\right)$ for all $p, e_{p^{*}}\left(k_{i} \alpha\right) \in G_{n}\left(X_{p}\right)$. So if we can show the result for $\alpha$ of order $p^{m}, p$ any prime, then each $k_{i} \alpha$ will be in $G_{n}(X)$ and thus $\alpha \in G_{n}(X)$.

We assume then that $\alpha$ has order $p^{m}$ for $p$ prime and localize at this p. Consider the following diagram:

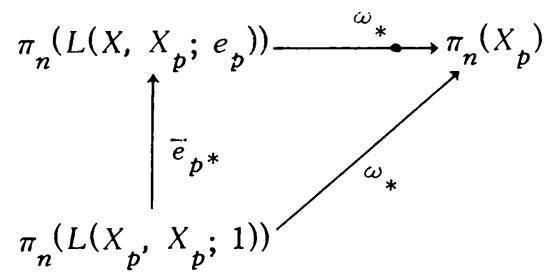

where $\bar{e}_{p}(f)=f e_{p}$. From this commutative diagram and the definition of $e_{p^{*}}(\alpha) \in G_{n}\left(X_{p}\right)$ it follows that there is an $\tilde{\alpha} \in \pi_{n}\left(L\left(X, X_{p} ; e_{p}\right)\right)$ such that $\omega_{*}(\tilde{\alpha})=e_{p^{*}}(\alpha)$. Let

$$
\bar{\phi}_{p}: \pi_{n}\left(L\left(X, X ; 1_{X}\right)\right) \otimes \dot{Q}_{p} \rightarrow \pi_{n}\left(L\left(X, X{ }_{p} ; e_{p}\right)\right)
$$

be the isomorphism guaranteed by Theorem 2.1. There is an element

$$
\sum\left(\alpha_{i} \otimes\left(k_{i} / q_{i}\right)\right) \in \pi_{n}\left(L\left(X, X ; 1_{X}\right)\right) \otimes Q_{p}
$$

such that $\bar{\phi}_{p}\left(\Sigma\left(\alpha_{i} \otimes\left(k_{i} / q_{i}\right)\right)\right)=\tilde{\alpha}$. If $q=\prod q_{i}$ and $\bar{q}_{i}=\prod_{j \neq i} q_{j}$ then

$$
\begin{aligned}
\sum\left(\alpha_{i} \otimes\left(k_{i} / q_{i}\right)\right) & =\sum\left(\alpha_{i} \otimes \bar{q}_{i} k_{i} / q\right) \\
& =\sum\left(\bar{q}_{i} k_{i} \alpha_{i} \otimes(1 / q)\right)=\left(\sum \bar{q}_{i} k_{i} \alpha_{i}\right) \otimes(1 / q) .
\end{aligned}
$$

Note that $p \nmid q_{i}$ for each $i$, hence $p \ell^{\prime} q$. Thus there is an element

$$
\beta \otimes(1 / q) \in \pi_{n}\left(L\left(X, X ; 1_{X}\right)\right) \otimes Q_{p}
$$

such that $\bar{\phi}_{p}(\beta \otimes(1 / q))=\tilde{\alpha}$ and $p \nmid q$. Consider the following diagram: 


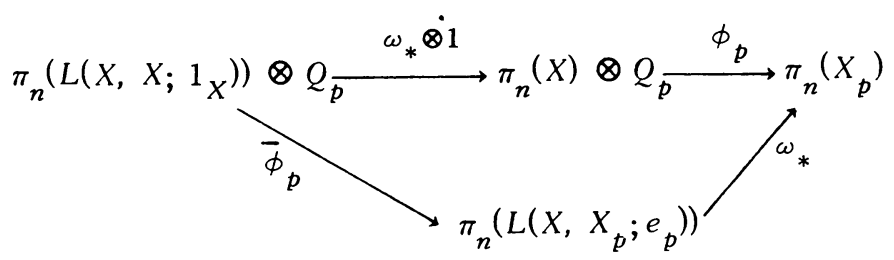

By commutativity

$$
\begin{aligned}
\phi_{p}\left(\omega_{*}(\beta) \otimes 1\right) & =\omega_{*}\left(\bar{\phi}_{p}(\beta \otimes 1)\right)=\omega_{*}\left(\bar{\phi}_{p}(q \beta \otimes(1 / q))\right) \\
& =\omega_{*}(q \alpha)=e_{p^{*}}(q \tilde{\alpha})
\end{aligned}
$$

But $e_{p^{*}}(q \alpha)=\phi_{p}(q \alpha \otimes 1)$, so $\omega_{*}(\beta) \otimes 1=q \alpha \otimes 1$ since $\phi_{p}$ is an isomorphism. But then $\omega_{*}(\beta)=q \alpha+\gamma$ where $\gamma$ has order $q^{\prime}$ and $p \nmid q^{\prime}$, so $\omega_{*}\left(q^{\prime} \beta\right)=q^{\prime} q \alpha$. Since $p^{m}$ and $q^{\prime} q$ are relatively prime there are integers $r$ and $s$ such that $r p^{m}+s q^{\prime} q=1$. Thus $\omega_{*}\left(s q^{\prime} \beta\right)=s q^{\prime} q \alpha=\alpha-r p^{m} \alpha=$ $\alpha$ and $\alpha \in G_{n}(X)$.

Case 2. $a$ of infinite order. Localizing at 0 the above argument yields an element

$$
\beta \otimes(1 / q) \in \pi_{n}\left(L\left(X, X ; 1_{X}\right)\right) \otimes Q_{0}
$$

such that $\omega_{*}(\beta) \otimes(1 / q)=\alpha \otimes 1$, and as above $\omega_{*}\left(q^{\prime} \beta\right)=q^{\prime} q \alpha$ for some nonzero integer $q^{\prime}$. Thus there are nonzero multiples of $\alpha$ in $G_{n}(X)$. Let $\bar{q}$ be the least positive integer such that $\bar{q} \alpha \in G_{n}(X)$. If $\bar{q} \neq 1$ let $p$ be a prime factor of $\bar{q}$. Localizing at this $p$ we obtain an element $\beta^{\prime} \epsilon$ $\pi_{n}\left(L\left(X, X ; 1_{X}\right)\right)$ and a $q^{\prime \prime}$ where $p X q^{\prime \prime}$ such that $\omega_{*}\left(\beta^{\prime}\right)=q^{\prime \prime} \alpha$. But then $q^{\prime \prime} \alpha$ is in the subgroup generated by $\bar{q} \alpha$ so $q^{\prime \prime}$ is a multiple of $\bar{q}$. This is a contradiction since $p$ is a factor of $\bar{q}$ but not of $q$ ". Thus $\bar{q}=1$ and $\alpha \in G_{n}(X)$.

During the proof we also obtained the following useful corollary.

Corollary 2.4. If $e_{p} *(\alpha) \in G_{n}\left(X_{p}\right)$ then there is a $q$ such that $p \backslash q$ and $q \alpha \in G_{n}(X)$.

Corollary 2.5. $G_{n}\left(X_{p}\right) \cong G_{n}(X)_{p}$.

Proof. We need only show that $\phi_{p}: \pi_{n}(X) \otimes Q_{p} \rightarrow \pi_{n}\left(X_{p}\right)$ satisfies

$$
\operatorname{Im} \phi_{p} \mid G_{n}(X) \otimes Q_{p}=G_{n}\left(X_{p}\right) .
$$

Say $\alpha \otimes(1 / q) \in G_{n}(X) \otimes Q_{p}$ (we can assume any element in $G_{n}(X) \otimes Q_{p}$ has this form by the technique used in 2.3). Let $F: X \times S^{n} \rightarrow X$ be an 
affiliated map for $\alpha$ and $g: S^{n} \rightarrow S_{p}^{n}$ a map corresponding to $1 / q \in \pi_{n}\left(S_{p}^{n}\right) \cong$ $Q_{p}$. Then the composition

$$
X_{p} \times S^{n \stackrel{1 \times g}{\longrightarrow}} X_{p} \times S_{p}^{\stackrel{F_{p}}{\longrightarrow}} X_{p}
$$

is an affiliated map for $\phi_{p}(\alpha \otimes(1 / q))$ and

$$
\operatorname{Im} \phi_{p} \mid G_{n}(X) \otimes Q_{p} \subset G_{n}\left(X_{p}\right) .
$$

Now say $\tilde{\alpha} \in G_{n}\left(X_{p}\right)$ and $\alpha \otimes(1 / q)=\phi_{p}^{-1}(\tilde{\alpha})$. Then $e_{p^{*}}(\alpha) \otimes 1=q \tilde{\alpha} \otimes 1$ and $e_{p^{*}}(\alpha)-q \tilde{\alpha}$ is of order $q^{\prime}$ where $p \nmid q^{\prime}$. Thus $e_{p^{*}}\left(q^{\prime} a\right)=q^{\prime} q \tilde{\alpha} \epsilon$ $G_{n}\left(X_{p}\right)$. By Corollary 2.5 there is a $q^{\prime \prime}$ such that $p \chi^{\prime} q^{\prime \prime}$ and $q^{\prime \prime} q^{\prime} \alpha \in G_{n}(X)$. Then

$q^{\prime \prime} q^{\prime} \alpha \otimes \frac{1}{q^{\prime \prime} q^{\prime} q} \in G_{n}(X) \otimes Q_{p}$ and $\phi_{p}\left(q^{\prime \prime} q^{\prime} \alpha \otimes \frac{1}{q^{\prime \prime} q^{\prime} q}\right)=\phi_{p}\left(\alpha \otimes \frac{1}{q}\right)=\tilde{\alpha}$, so $\tilde{a} \in \operatorname{Im} \phi_{p} \mid G_{n}(X) \otimes Q_{p}$. Thus $\phi_{p} \mid G_{n}(X) \otimes Q_{p}$ is onto $G_{n}\left(X_{p}\right)$.

Corollary 2.6. $X$ is a G-space if and only if $X_{p}$ is a G-space for all $p$.

Proof. By definition $X$ is a $G$-space if and only if $G_{n}(X)=\pi_{n}(X)$ for all $n$. If $X$ is a $G$-space, $X_{p}$ is a $G$-space for all $p$ by Corollary 2.5. If $X_{p}$ is a $G$-space for all $p$ then $X$ is a $G$-space by Theorem 2.3.

The following application illustrates how these results can be used.

Corollary 2.7. If $G_{n}(X)$ is torsion without p-torsion, any fibration $f: E \rightarrow S^{n+1}$ with fiber $X_{p}$ admits a cross section.

Proof. In this case $G_{n}(X)_{p}=0$ so by Corollary $2.5 G_{n}\left(X_{p}\right)=0$ and the result follows from Corollary 2.7 in [1]

For example, if $V$ is the real Stiefel manifold $V_{n_{v} k}$ where $n-k=1$ or $3, k>1$, any fibration over $S^{n-k+1}$ with fiber $V_{p}, p$ an odd prime, admits a cross section. This follows from Corollary 2.7 and results in [4].

Corollary 2.4 also provides a shortened proof of Proposition 3 in [2] with simply connected replaced by simple. Let $\mathcal{C}$ be a Serre class of Abelian groups. Haslam defines a $G$-space mod $C$ to be a space $X$ such that $\pi_{n}(X) / G_{n}(X) \in \mathcal{C}$. A space will be called an $H$-space $\bmod \mathcal{C}$ if there is a map $\mu: X \times X \rightarrow X$ which, when restricted to each factor, induces a $C$ isomorphism in homotopy. Let $C_{p}$ be the class of finite Abelian groups having no element with order a positive power of $p$; let $C_{0}=\mathcal{F}$ be the class of finite Abelian groups. 
Theorem 2.8 (Haslam). If $X$ is an $H$-space mod $C_{p}$, prime or 0 , then $X$ is a G-space $\bmod \mathcal{C}_{p}$.

Proof. If $X$ is an $H$-space mod $C_{p}$ then it can be shown that $X_{p}$ is an $H$-space and hence a $G$-space. For every $\alpha \in \pi_{n}(X), e_{p}(\alpha) \in G_{n}\left(X_{p}\right)$ and by Corollary 2.4 there is a $q$ where $p \nmid q$ such that $q \alpha \in G_{n}(X)$. Thus $X$ is a $G$-space $\bmod \mathcal{C}_{p}$.

For $\mathcal{C}_{0}$ Haslam has obtained a converse to this theorem [2]. For $\mathcal{C}_{p}$, $p$ prime, the converse is false in general but the simply connected case is still open.

\section{REFERENCES}

1. D. H. Gottlieb, Evaluation subgroups of homotopy groups, Amer. J. Math. 91 (1969), 729-756. MR 43 \#1181.

2. H. B. Haslam, $G$-spaces $\bmod F$ and $H$-spaces $\bmod F$, Duke Math. J. 38 (1971), 671-679. MR 44 \# 4742.

3. P. J. Hilton, G. Mislin and J. Roitberg, Homotopical localization (unpublished).

4. G. E. Lang, Evaluation subgroups of factor spaces, Pacific J. Math 42 (1972), 701-709. MR $47 \# 2595$.

5. D. Sullivan, Geometric topology. I: Localization, periodicity and Galois symmetry, M. I. T., June 1970 (mimeo).

DEPARTMENT OF MATHEMATICS, FAIRFIELD UNIVERSITY, F AIRFIELD, CONNECTICUT 06430 